\title{
Comment on "Liquid ${ }^{4} \mathrm{He}$ : Contributions to First Principles Theory. II. Quantized Vortices and the Lambda Transition"
}

\author{
Gary A. Williams
}

Received: 4 June 2009 / Accepted: 24 June 2009 / Published online: 8 July 2009

(C) The Author(s) 2009. This article is published with open access at Springerlink.com

\begin{abstract}
A comment on the paper "Liquid ${ }^{4} \mathrm{He}$ : Contributions to First Principles Theory. II. Quantized Vortices and the Lambda Transition" by H.W. Jackson (J. Low Temp. Phys. 155:1, 2009).
\end{abstract}

Keywords Vortex loops $\cdot$ Superfluid transition

PACS $67.25 . \mathrm{dj} \cdot 67.25 . \mathrm{dk} \cdot 67.25 . \mathrm{bh} \cdot 67.25 . \mathrm{dg}$

\section{Introduction}

In a recent paper H.W. Jackson [1] has claimed there is an error in my original paper on the role of vortex loops at the superfluid phase transition [2] which invalidates the theory. I thank the author for pointing out the sign error, but note that this error only crept in during the write-up for publication, and not in the actual calculation. In fact the result that the loop polarizability (Eq. 2 of Ref. [2]) varies as $R^{4}$ was already well known in the literature [3]. The subsequent calculation using this expression for the polarizability has nothing whatsoever to do with the sign error in the text; Jackson is completely wrong in claiming that this invalidates the theory. The same recursion relations found in that paper (Eqs. 9) were later confirmed to within unimportant constants by Shenoy [4], using a rather different approach starting directly from the XY model Hamiltonian and not involving any calculation of the polarizability.

I disagree with many of the other claims in the Jackson paper, and in particular the claim that the critical fluctuations at the lambda point arise from a previously

Work supported by the National Science Foundation, Grant DMR 05-48521.

G.A. Williams (凶)

Department of Physics and Astronomy, University of California, Los Angeles, CA 90095, USA

e-mail: gaw@ucla.edu 
undiscovered new kind of sound in bulk superfluid helium, which the author calls "isothermal fourth sound." This is a mode where the normal fluid velocity is mysteriously clamped to zero in the bulk liquid, temperature oscillations do not occur, and the speed of the mode is the square root of the superfluid fraction times the first sound speed. How the normal fluid could be clamped in the thermodynamic limit of an infinite system is not apparent. Walls will not clamp the normal fluid at distances exceeding a viscous penetration depth, about a micrometer at $\mathrm{kHz}$ frequencies, and at larger distances the normal fluid will be perfectly free to move. I do not believe that such a mode can exist in the bulk, and there is certainly no experimental evidence for it. The mode is a pressure wave that will couple very strongly to pressure sensors, and should have been easily observable in any acoustic first-sound cell ever constructed.

Jackson claims in Appendix C of [1] that the clamping of the normal fluid and the lack of temperature fluctuations in the putative "isothermal fourth sound" is a consequence of the two-fluid equations in "thermodynamic equilibrium." He then goes on to question the correctness of experimental measurements of first and second sound near the lambda point, because "the liquid He II is excited by external means and therefore was not in thermodynamic equilibrium." I do not believe this to be the case, and it is very easy to cite a specific counter-example: Brillouin light-scattering measurements $[5,6]$ allow the measurement of thermally excited propagating modes with no drive inputs necessary at all. The only modes observed in these studies (certainly at thermal equilibrium) are the usual first and second sound modes. These results are in full agreement with the known sound modes, even very close to the lambda point, and no additional modes could be detected. Both first and second sound involve nonzero normal fluid velocities, and second sound is certainly accompanied by temperature oscillations, proving that such fluctuations are present even in "thermal equilibrium." The "isothermal fourth sound" mode claimed by Jackson simply does not exist in bulk superfluid helium.

Open Access This article is distributed under the terms of the Creative Commons Attribution Noncommercial License which permits any noncommercial use, distribution, and reproduction in any medium, provided the original author(s) and source are credited.

\section{References}

1. H.W. Jackson, J. Low Temp. Phys. 155, 1 (2009)

2. G.A. Williams, Phys. Rev. Lett. 59, 1926 (1987)

3. W. Helfrich, J. Phys. (Paris) 39, 1199 (1978)

4. S.R. Shenoy, Phys. Rev. B 40, 5056 (1989)

5. G. Winterling, F.S. Holmes, T.J. Greytak, Phys. Rev. Lett. 30, 427 (1973)

6. W.F. Vinen, D.L. Hurd, Adv. Phys. 27, 533 (1978) 